\title{
Homothetic Motion and Surfaces with Pointwise 1-Type Gauss Map in $\mathbb{E}^{4}$
}

\author{
Ferdağ Kahraman Aksoyak, Yusuf Yaylı
}

Ferdağ Kahraman Aksoyak: Ahi Evran University, Division of Elementary Mathematics Education, Kirsehir, Turkey, e-mail:ferdag.aksoyak@ahievran.edu.tr,

Yusuf Yaylı: Ankara University, Department of Mathematics, Ankara, Turkey, email:yayli@science.ankara.edu.tr

\begin{abstract}
In this paper, we determine a surface $M$ by means of homothetic motion in $\mathbb{E}^{4}$ and we give necessary and sufficient conditions for flat surface $M$ with flat normal bundle to have pointwise 1-type Gauss map. Also, we show that flat surface $M$ with flat normal bundle which have pointwise 1-type Gauss map of the first kind is a Clifford Torus. Morever, we obtain a characterization of minimal surface $M$ with pointwise 1-type Gauss map.
\end{abstract}

Keywords. Homothetic motion · submanifolds . Gauss map · Pointwise 1-type Gauss map.

MSC 2010 Classification. Primary: 53B25; Secondary:53C40.

\section{$1 \quad$ INTRODUCTION}

A submanifold $M$ of a Euclidean space $\mathbb{E}^{m}$ is said to be of finite type if its position vector $x$ can be expressed as a finite sum of eigenvectors of the Laplacian $\Delta$ of $M$, that is, $x=x_{0}+x_{1}+\ldots+x_{k}$, where $x_{0}$ is a constant map, $x_{1}, \ldots, x_{k}$ are non-constant maps such that $\Delta x_{i}=\lambda_{i} x_{i}, \lambda_{i} \in \mathbb{R}, i=1,2, \ldots, k$. If $\lambda_{1}, \lambda_{2}, \ldots, \lambda_{k}$ are all different, then $M$ is said to be of $k$-type. This definition was similarly extended to differentiable maps, in particular, to Gauss maps of submanifolds [3].

If a submanifold $M$ of a Euclidean space has 1-type Gauss map $G$, then $G$ satisfies $\Delta G=\lambda(G+C)$ for some $\lambda \in \mathbb{R}$ and some constant vector $C$. Chen and Piccinni made a general study on compact submanifolds of Euclidean spaces with finite type Gauss map and they proved that a compact hypersurface $M$ of $\mathbb{E}^{n+1}$ has 1-type Gauss map if and only if $M$ is a hypersphere in $\mathbb{E}^{n+1}[3]$.

Hovewer, the Laplacian of the Gauss map of some typical well known surfaces such as a helicoid, a catenoid and a right cone in Euclidean 3 -space $\mathbb{E}^{3}$ take a some what different form, namely,

$$
\Delta G=f(G+C)
$$


for some smooth function $f$ on $M$ and some constant vector $C$. A submanifold $M$ of a Euclidean space $\mathbb{E}^{m}$ is said to have pointwise 1-type Gauss map if its Gauss map satisfies (1) for some smooth function $f$ on $M$ and some constant vector $C$. A submanifold with pointwise 1-type Gauss map is said to be of the first kind if the vector $C$ in (1) is zero vector. Otherwise, the pointwise 1-type Gauss map is said to be of the second kind. A pointwise 1-type Gauss map is called proper if the function $f$ given by (1.1) is non-constant. Non-proper pointwise 1-type Gauss map is just usual 1-type Gauss map.

Surfaces in Euclidean space with pointwise 1-type Gauss map were recently studied in [4], [5], [6]. Also Dursun and Turgay in [7] gave all general rotational surfaces in $\mathbb{E}^{4}$ with proper pointwise 1-type Gauss map of the first kind and classified minimal rotational surfaces with proper pointwise 1-type Gauss map of the second kind. Arslan et al. in [1] investigated rotational embedded surface with pointwise 1-type Gauss map. Arslan at el. in [2] gave necessary and sufficent conditions for Vranceanu rotation surface to have pointwise 1-type Gauss map. Yoon in [8] showed that flat Vranceanu rotation surface with pointwise 1-type Gauss map is a Clifford torus.

In this paper, we determine a surface $M$ by means of homothetic motion in $\mathbb{E}^{4}$ and we give necessary and sufficient conditions for flat surface $\mathrm{M}$ with flat normal bundle to have pointwise 1-type Gauss map. We show that flat surface with flat normal bundle which has pointwise 1-type Gauss map of the first kind is a Clifford Torus. Morever we obtain a characterization of minimal surface $M$ with pointwise 1-type Gauss map.

\section{PRELIMINARIES}

Let $M$ be an oriented $n$-dimensional submanifold in $m$-dimensional Euclidean space $\mathbb{E}^{m}$. Let $e_{1}, \ldots, e_{n}, e_{n+1}, \ldots, e_{m}$ be an oriented local orthonormal frame in $\mathbb{E}^{m}$ such that $e_{1}, \ldots, e_{n}$ are tangent to $M$ and $e_{n+1}, \ldots, e_{m}$ normal to $M$. We use the following convention on the ranges of indices: $1 \leq i, j, k, \ldots \leq n, n+1 \leq$ $r, s, t, \ldots \leq m, 1 \leq A, B, C, \ldots \leq m$.

Let $\tilde{\nabla}$ be the Levi-Civita connection of $\mathbb{E}^{m}$ and $\nabla$ the induced connection on $M$. Let $\omega_{A}$ be the dual-1 form of $e_{A}$ defined by $\omega_{A}\left(e_{B}\right)=\delta_{A B}$. Also, the connection forms $\omega_{A B}$ are defined by

$$
d e_{A}=\sum_{B} \omega_{A B} e_{B}, \quad \omega_{A B}+\omega_{B A}=0 .
$$

Then we have

$$
\tilde{\nabla}_{e_{k}} e_{i}=\sum_{j=1}^{n} \omega_{i j}\left(e_{k}\right) e_{j}+\sum_{r=n+1}^{m} h_{i k}^{r} e_{r}
$$

and

$$
\tilde{\nabla}_{e_{k}} e_{s}=-A_{s}\left(e_{k}\right)+D_{e_{k}} e_{s}, \quad D_{e_{k}} e_{s}=\sum_{r=n+1}^{m} \omega_{s r}\left(e_{k}\right) e_{r},
$$


where $D$ is the normal connection, $h_{i k}^{r}$ the coefficients of the second fundamental form $h$ and $A_{s}$ the Weingarten map in the direction $e_{s}$.

For any real function $f$ on $M$, the Laplacian of $f$ is defined by

$$
\Delta f=-\sum_{i}\left(\tilde{\nabla}_{e_{i}} \tilde{\nabla}_{e_{i}} f-\tilde{\nabla}_{\nabla_{e_{i}}^{e_{i}}} f\right) .
$$

The mean curvature vector $H$ and Gaussian curvature $K$ are defined by

$$
H=\frac{1}{n} \sum_{r, i} h_{i i}^{r} e_{r}
$$

and

$$
K=\sum_{s=n+1}^{m}\left(h_{11}^{s} h_{22}^{s}-h_{12}^{s} h_{21}^{s}\right) .
$$

Also normal curvature tensor $R^{D}$ of $M$ in $\mathbb{E}^{m}$ is given by

$$
R^{D}\left(e_{j}, e_{k} ; e_{r}, e_{s}\right)=\sum_{i=1}^{n}\left(h_{i k}^{r} h_{i j}^{s}-h_{i j}^{r} h_{i k}^{s}\right) .
$$

Let us now define the Gauss map $G$ of a submanifold $M$ into $G(n, m)$ in $\wedge^{n} \mathbb{E}^{m}$, where $G(n, m)$ is the Grassmannian manifold consisting of all oriented $n$-planes through the origin of $\mathbb{E}^{m}$ and $\wedge^{n} \mathbb{E}^{m}$ is the vector space obtained by the exterior product of $n$ vectors in $\mathbb{E}^{m}$. In a natural way, we can identify $\wedge^{n} \mathbb{E}^{m}$ with some Euclidean space $\mathbb{E}^{N}$ where $N=\left(\begin{array}{c}m \\ n\end{array}\right)$. The map $G: M \rightarrow$ $G(n, m) \subset E^{N}$ defined by $G(p)=\left(e_{1} \wedge \ldots \wedge e_{n}\right)(p)$ is called the Gauss map of $M$, that is, a smooth map which carries a point $p$ in $M$ into the oriented $n$-plane through the origin of $\mathbb{E}^{m}$ obtained from parallel translation of the tangent space of $M$ at $p$ in $\mathbb{E}^{m}$.

The Laplacian of the Gauss map $G$ for an $n$-dimensional submanifold $M$ of Euclidean space $\mathbb{E}^{m}$ was given by

Lemma 2.1. (See [3]) Let $x: M \rightarrow \mathbb{E}^{m}$ be an isometric immersion of an oriented n-dimensional Riemannian manifold $M$ into $\mathbb{E}^{m}$. Then the Laplacian of the Gauss map $G: M \rightarrow G(n, m) \subset \wedge^{n} \mathbb{E}^{m}$ is given by

$$
\begin{aligned}
\Delta G= & -n \sum_{i} e_{1} \wedge \ldots \wedge D_{e_{i}} H \wedge \ldots \wedge e_{n} \\
& +R^{D}\left(e_{j}, e_{k} ; e_{r}, e_{s}\right) e_{1} \wedge \ldots \wedge e_{s}^{k}{ }^{t h} \wedge \ldots \wedge e_{r}^{j t h} \wedge \ldots \wedge e_{n}+\|h\|^{2} G .
\end{aligned}
$$




\section{Homothetic Motion and Surfaces with Pointwise 1-Type Gauss MaP}

In this section, we define a surface by using the homothetic motion as follows:

$$
f(t, s)=h(t)\left(\begin{array}{cccc}
\cos t & -\sin t & 0 & 0 \\
\sin t & \cos t & 0 & 0 \\
0 & 0 & \cos t & -\sin t \\
0 & 0 & \sin t & \cos t
\end{array}\right)\left(\begin{array}{l}
\alpha_{1}(s) \\
\alpha_{2}(s) \\
\alpha_{3}(s) \\
\alpha_{4}(s)
\end{array}\right)+\left(\begin{array}{c}
C_{1}(t) \\
C_{2}(t) \\
C_{3}(t) \\
C_{4}(t)
\end{array}\right)
$$

where $h(t)$ is the homothetic scale of the motion, $C(t)=\left(C_{1}(t), C_{2}(t), C_{3}(t), C_{4}(t)\right)$ is the translation vector and $\alpha(s)=\left(\alpha_{1}(s), \alpha_{2}(s), \alpha_{3}(s), \alpha_{4}(s)\right)$ is a profile curve. If we choose the profile curve $\alpha$ as $\alpha(s)=(u(s) \cos s, 0, u(s) \sin s, 0)$ and the translation vector $C(t)=\overrightarrow{0}$ in (3.1), we obtain the surface $M$ as follows:

$f(s, t)=(u(s) h(t) \cos s \cos t, u(s) h(t) \cos s \sin t, u(s) h(t) \sin s \cos t, u(s) h(t) \sin s \sin t)$

Let $M$ be a surface in $\mathbb{E}^{4}$ given by the parametrization (3.2). The tangent vectors of $f(s, t)$ can be easily computed as

$$
\begin{aligned}
& \vec{v}_{1}=\frac{\partial f}{\partial t}=\left(A_{1} B_{1}^{\prime}, A_{1} B_{2}^{\prime}, A_{2} B_{1}^{\prime}, A_{2} B_{2}^{\prime}\right) \\
& \vec{v}_{2}=\frac{\partial f}{\partial s}=\left(\dot{A}_{1} B_{1}, \dot{A}_{1} B_{2}, \dot{A}_{2} B_{1}, \dot{A}_{2} B_{2}\right)
\end{aligned}
$$

and a basis of the normal space of $f(s, t)$ can be given as follows:

$$
\begin{aligned}
& \vec{v}_{3}=\left(-A_{2} B_{2}, A_{2} B_{1}, A_{1} B_{2},-A_{1} B_{1}\right), \\
& \vec{v}_{4}=\left(-\dot{A}_{2} B_{2}^{\prime}, \dot{A}_{2} B_{1}^{\prime}, \dot{A}_{1} B_{2}^{\prime},-\dot{A}_{1} B_{1}^{\prime}\right),
\end{aligned}
$$

where

$$
\begin{aligned}
& A_{1}=u(s) \cos s, \quad A_{2}=u(s) \sin s \\
& B_{1}=h(t) \cos t, \quad B_{2}=h(t) \sin t
\end{aligned}
$$

and $\dot{A}_{i}=\frac{\partial A_{i}}{\partial s}$ for $i=1,2$ and $B_{j}^{\prime}=\frac{\partial B_{j}}{\partial t} j=1,2$. By using Gramm-Schmidth orthonormalization, the orthonormal vectors of tangent and normal spaces of $M$ are obtained, respectively, by

$$
\begin{aligned}
& e_{1}=\frac{1}{\sqrt{v_{11}}} \vec{v}_{1}, \\
& e_{2}=\frac{1}{\sqrt{\left|v_{11}\left(v_{11} v_{22}-v_{12}^{2}\right)\right|}}\left(v_{11} \vec{v}_{2}-v_{12} \vec{v}_{1}\right)
\end{aligned}
$$


and

$$
\begin{aligned}
& e_{3}=\frac{1}{\sqrt{v_{33}}} \vec{v}_{3}, \\
& e_{4}=\frac{1}{\sqrt{\left|v_{33}\left(v_{33} v_{44}-v_{34}^{2}\right)\right|}}\left(v_{33} \vec{v}_{4}-v_{34} \vec{v}_{3}\right),
\end{aligned}
$$

where

$$
\begin{aligned}
& v_{11}=\left\langle\vec{v}_{1}, \vec{v}_{1}\right\rangle=u^{2}(s)\left(h^{2}(t)+\left(h^{\prime}(t)\right)^{2}\right), \\
& v_{12}=\left\langle\vec{v}_{1}, \vec{v}_{2}\right\rangle=u(s) \dot{u}(s) h(t) h^{\prime}(t), \\
& v_{22}=\left\langle\vec{v}_{2}, \vec{v}_{2}\right\rangle=\left(u^{2}(s)+(\dot{u}(s))^{2}\right) h^{2}(t), \\
& v_{33}=\left\langle\vec{v}_{3}, \vec{v}_{3}\right\rangle=u^{2}(s) h^{2}(t), \\
& v_{34}=\left\langle\vec{v}_{3}, \vec{v}_{4}\right\rangle=u(s) \dot{u}(s) h(t) h^{\prime}(t), \\
& v_{44}=\left\langle\vec{v}_{4}, \vec{v}_{4}\right\rangle=\left(u^{2}(s)+(\dot{u}(s))^{2}\right)\left(h^{2}(t)+\left(h^{\prime}(t)\right)^{2}\right) .
\end{aligned}
$$

Hence, $\left\{e_{1}, e_{2}, e_{3}, e_{4}\right\}$ is orthonormal moving frame on $M$. Then we have the dual 1-forms as:

$$
\begin{aligned}
& \omega_{1}=\frac{\dot{u} h h^{\prime}}{\left(h^{2}+\left(h^{\prime}\right)^{2}\right)^{\frac{1}{2}}} d s+\frac{u\left(h^{2}+\left(h^{\prime}\right)^{2}\right)}{\left(h^{2}+\left(h^{\prime}\right)^{2}\right)^{\frac{1}{2}}} d t \\
& \omega_{2}=\frac{h\left(u^{2} h^{2}+u^{2}\left(h^{\prime}\right)^{2}+(\dot{u})^{2} h^{2}\right)^{\frac{1}{2}}}{\left(h^{2}+\left(h^{\prime}\right)^{2}\right)^{\frac{1}{2}}} d s
\end{aligned}
$$

By a direct computation we have components of the second fundamental form and the connection forms as:

$$
\begin{gathered}
h_{11}^{3}=0, \quad h_{12}^{3}=-\frac{1}{W^{\frac{1}{2}}}, \quad h_{22}^{3}=2 \frac{\dot{u} h^{\prime}}{W} \\
h_{11}^{4}=\frac{\left(2\left(h^{\prime}\right)^{2}-h h^{\prime \prime}+h^{2}\right)}{\left(h^{2}+\left(h^{\prime}\right)^{2}\right) W^{\frac{1}{2}}}, \\
h_{12}^{4}=\frac{\dot{u} h^{\prime}\left(h h^{\prime \prime}-\left(h^{\prime}\right)^{2}\right)}{\left(h^{2}+\left(h^{\prime}\right)^{2}\right) W}, \\
h_{22}^{4}=\frac{\left(2(\dot{u})^{2}-u \ddot{u}+u^{2}\right)\left(h^{2}+\left(h^{\prime}\right)^{2}\right)^{2}-(\dot{u})^{2}\left(h^{\prime}\right)^{2}\left(h h^{\prime \prime}+h^{2}\right)}{\left(h^{2}+\left(h^{\prime}\right)^{2}\right)^{\frac{3}{2}}}
\end{gathered}
$$


and

$$
\begin{aligned}
\omega_{12}= & -\frac{\dot{u} h\left(2\left(h^{\prime}\right)^{2}-h h^{\prime \prime}+h^{2}\right)}{u\left(h^{2}+\left(h^{\prime}\right)^{2}\right)^{\frac{3}{2}} W^{\frac{1}{2}}} \omega_{1} \\
& +\frac{u^{2} h^{\prime}\left(h^{2}+\left(h^{\prime}\right)^{2}\right)^{2}+(\dot{u})^{2} h^{2} h^{\prime}\left(2\left(h^{\prime}\right)^{2}-h h^{\prime \prime}+h^{2}\right)}{u h\left(h^{2}+\left(h^{\prime}\right)^{2}\right)^{\frac{3}{2}} W} \omega_{2}, \\
\omega_{34}= & \frac{\dot{u} h}{u\left(h^{2}+\left(h^{\prime}\right)^{2}\right)^{\frac{1}{2}} W^{\frac{1}{2}}} \omega_{1}+\frac{h^{\prime}\left(u^{2} h^{2}+u^{2}\left(h^{\prime}\right)^{2}-(\dot{u})^{2} h^{2}\right)}{u h\left(h^{2}+\left(h^{\prime}\right)^{2}\right)^{\frac{1}{2}} W} \omega_{2},
\end{aligned}
$$

where $W=u^{2} h^{2}+u^{2}\left(h^{\prime}\right)^{2}+(\dot{u})^{2} h^{2}$.

Proposition 3.1. Let $M$ be the surface given by the parameterization (3.2). The Gaussian curvature and the normal bundle curvature of $M$ are given, respectively, by

$$
K=\frac{\left(2\left(h^{\prime}\right)^{2}-h h^{\prime \prime}+h^{2}\right)\left(2(\dot{u})^{2}-u \ddot{u}+u^{2}\right)-\left(h^{2}+\left(h^{\prime}\right)^{2}\right)\left(u^{2}+(\dot{u})^{2}\right)}{W^{2}}
$$

and

$$
R^{D}=\frac{\left(2(\dot{u})^{2}-u \ddot{u}+u^{2}\right)\left(h^{2}+\left(h^{\prime}\right)^{2}\right)-\left(2\left(h^{\prime}\right)^{2}-h h^{\prime \prime}+h^{2}\right)\left(u^{2}+(\dot{u})^{2}\right)}{W^{2}}
$$

Proof. By using (2.3), (2.4), (3.3) and (3.4), we obtain (3.6) and (3.7).

Corollary 3.2. Let $M$ be the surface given by the parameterization (3.2). $M$ is a flat surface with flat normal bundle if and only if it is parameterized by

$$
f(t, s)=a_{1} a_{2} e^{k_{1} t+k_{2} s}(\cos s \cos t, \cos s \sin t, \sin s \cos t, \sin s \sin t)
$$

or

$f(t, s)=\frac{c_{1} c_{2}}{\sqrt{\left|\cos \left(2 t+b_{1}\right)\right|} \sqrt{\left|\cos \left(2 s+b_{2}\right)\right|}}(\cos s \cos t, \cos s \sin t, \sin s \cos t, \sin s \sin t)$

Proof. Let $M$ be a flat surface with flat normal bundle. Then both $K=0$ and $R^{D}=0$. From (3.6), we have

$$
\frac{2\left(h^{\prime}\right)^{2}-h h^{\prime \prime}+h^{2}}{h^{2}+\left(h^{\prime}\right)^{2}} \cdot \frac{2(\dot{u})^{2}-u \ddot{u}+u^{2}}{u^{2}+(\dot{u})^{2}}=1
$$


and from (3.7), we get

$$
\frac{2\left(h^{\prime}\right)^{2}-h h^{\prime \prime}+h^{2}}{h^{2}+\left(h^{\prime}\right)^{2}}=\frac{2(\dot{u})^{2}-u \ddot{u}+u^{2}}{u^{2}(s)+(\dot{u})^{2}} .
$$

By combining (3.10) and (3.11) and solving these differential equations we obtain

$$
h(t)=a_{1} e^{k_{1} t} \text { and } u(s)=a_{2} e^{k_{2} s}
$$

or

$$
h(t)=\frac{c_{1}}{\sqrt{\left|\cos \left(2 t+b_{1}\right)\right|}} \text { and } u(s)=\frac{c_{2}}{\sqrt{\left|\cos \left(2 s+b_{2}\right)\right|}},
$$

where $a_{1}, a_{2}, b_{1}, b_{2}, c_{1}, c_{2}, k_{1}$ and $k_{2}$ are real constants.

Remark 3.3. The surface $M$ given by the parameterization (3.2) can be considered as the tensor product surface of two Euclidean planar curves, that is, let $\alpha: \mathbb{R} \rightarrow \mathbb{R}^{2}, \alpha(s)=\left(\alpha_{1}(s), \alpha_{2}(s)\right)$ and $\beta: \mathbb{R} \rightarrow \mathbb{R}^{2}, \beta(t)=\left(\beta_{1}(t), \beta_{2}(t)\right)$ be two Euclidean planar curves. The tensor product surface $f(t, s)$ is defined by

$$
\begin{gathered}
f=\alpha \otimes \beta: \mathbb{R}^{2} \rightarrow \mathbb{R}^{4}, \\
f(t, s)=\left(\alpha_{1}(s) \beta_{1}(t), \alpha_{1}(s) \beta_{2}(t), \alpha_{2}(s) \beta_{1}(t), \alpha_{2}(s) \beta_{2}(t)\right) .
\end{gathered}
$$

In particular, for the curves $\alpha(s)=(u(s) \cos s, u(s) \sin s)$ and $\beta(t)=(h(t) \cos t, h(t) \sin t)$ the tensor product of them gives the surface $M$ given by the parameterization (3.2).

Theorem 3.4. (See [9]). A regular tensor product surface $x(s, t)=\alpha(s) \otimes \beta(t)$ of two curves $\alpha: \mathbb{R} \rightarrow \mathbb{R}^{2}, \alpha(s)=(u(s) \cos s, u(s) \sin s)$ or $\beta: \mathbb{R} \rightarrow \mathbb{R}^{2}, \beta(t)=$ $(h(t) \cos t, h(t) \sin t)$ is flat if and only if either

1. $\alpha$ or $\beta$ is a straight line through the origin.

2. $\alpha$ and $\beta$ are sinusoidal spirals, that is, the curves $\alpha$ and $\beta$ are parameterized by

$$
\begin{aligned}
& \alpha(s)=c_{1}\left|\cos \left((a+1) s+b_{1}\right)\right|^{-\frac{1}{a+1}}(\cos s, \sin s) \\
& \beta(t)=c_{2}\left|\cos \left(\left(\frac{1}{a}+1\right) t+b_{2}\right)\right|^{-\frac{1}{a}+1}(\cos t, \sin t)
\end{aligned}
$$

3. $\alpha$ and $\beta$ are logarithmic spirals, that is, the curves $\alpha$ and $\beta$ are parameterized by

$$
\alpha(s)=a_{1} e^{k_{1} s}(\cos s, \sin s) \text { and } \beta(t)=a_{2} e^{k_{2} t}(\cos t, \sin t)
$$

with $a_{1}, a_{2}, b_{1}, b_{2}, c_{1}, c_{2}, k_{1}$ and $k_{2}$ are real constants, $a_{1}, a_{2}, c_{1}, c_{2}>0$ and $a \neq$ -1 . 
Remark 3.5. In [8] Yoon studied Vranceanu surface parameterized by

$$
f(s, t)=(u(s) \cos s \cos t, u(s) \cos s \sin t, u(s) \sin s \cos t, u(s) \sin s \sin t) .
$$

He proved that flat Vranceanu surface in $E^{4}$ has pointwise 1-type Gauss map if and only if it is a Clifford torus. Also the normal bundle of flat Vranceanu surface is flat, too.

Now we investigate flat surface $M$ with flat normal bundle with pointwise 1-type Gauss map.

Theorem 3.6. Let $M$ be flat surface with flat normal bundle given by the parameterization (3.2). Then $M$ has pointwise 1-type Gauss map if and only if either

1. $M$ is a Clifford torus, that is, the product of two plane circles with same radius

2. It is the product of two logarithmic spirals which is parameterized by

$$
f(t, s)=e^{k(t \pm s)}(\cos s \cos t, \cos s \sin t, \sin s \cos t, \sin s \sin t)
$$

where $k$ is non zero real constant.

Proof. Firstly, we assume that the flat surface $M$ with flat normal bundle given by the parameterization (3.8) has pointwise 1-type Gauss map. If necessary, by an appropriate homothetic transformation we may assume that $a_{1}=a_{2}=1$. Then we have $h(t)=e^{k_{1} t}$ and $u(s)=e^{k_{2} s}$. By using (3.3), (3.4) and (3.5) we have components of the second fundamental form and the connection forms as:

$$
\begin{gathered}
h_{11}^{3}=0, \quad h_{12}^{3}=-\alpha(s, t), \quad h_{22}^{3}=a \alpha(s, t) \\
h_{11}^{4}=\alpha(s, t), \quad h_{12}^{4}=0, \quad h_{22}^{4}=\alpha(s, t)
\end{gathered}
$$

and

$$
\begin{aligned}
& \omega_{12}=b \alpha(s, t) \omega_{1}+c \alpha(s, t) \omega_{2}, \quad \omega_{13}=-\alpha(s, t) \omega_{2}, \quad \omega_{14}=\alpha(s, t) \omega_{1} \\
& \omega_{23}=-\alpha(s, t) \omega_{1}+a \alpha(s, t) \omega_{2} \quad \omega_{24}=\alpha(s, t) \omega_{2}, \quad \omega_{34}=-b \alpha(s, t) \omega_{1}+d \alpha(s, t) \omega_{2},
\end{aligned}
$$

By covariant differentiation with respect to $e_{1}$ and $e_{2}$ a straightforward calculation gives:

$$
\begin{aligned}
\tilde{\nabla}_{e_{1}} e_{1} & =b \alpha e_{2}+\alpha e_{4}, \\
\tilde{\nabla}_{e_{2}} e_{1} & =c \alpha e_{2}-\alpha e_{3}, \\
\tilde{\nabla}_{e_{1}} e_{2} & =-b \alpha e_{1}-\alpha e_{3}, \\
\tilde{\nabla}_{e_{2}} e_{2} & =-c \alpha e_{1}+a \alpha e_{3}+\alpha e_{4} \\
\tilde{\nabla}_{e_{1}} e_{3} & =\alpha e_{2}-b \alpha e_{4} \\
\tilde{\nabla}_{e_{2}} e_{3} & =\alpha e_{1}-a \alpha e_{2}+d \alpha e_{4} \\
\tilde{\nabla}_{e_{1}} e_{4} & =-\alpha e_{1}+b \alpha e_{3} \\
\tilde{\nabla}_{e_{2}} e_{4} & =-\alpha e_{2}-d \alpha e_{3}
\end{aligned}
$$


where

$$
\begin{aligned}
\alpha(s, t) & =\frac{1}{u(s) h(t)\left(1+k_{1}^{2}+k_{2}^{2}\right)^{\frac{1}{2}}}, a=\frac{2 k_{1} k_{2}}{\left(1+k_{1}^{2}+k_{2}^{2}\right)^{\frac{1}{2}}}, b=-\frac{k_{2}}{\left(1+k_{1}^{2}\right)^{\frac{1}{2}}}, \\
c & =\frac{k_{1}\left(1+k_{1}^{2}+k_{2}^{2}\right)^{\frac{1}{2}}}{\left(1+k_{1}^{2}\right)^{\frac{1}{2}}}, d=\frac{k_{1}\left(1+k_{1}^{2}-k_{2}^{2}\right)}{\left(1+k_{1}^{2}\right)^{\frac{1}{2}}\left(1+k_{1}^{2}+k_{2}^{2}\right)^{\frac{1}{2}}}
\end{aligned}
$$

By using (2.1) and (3.12) and after straight-forward computations, the Laplacian $\Delta G$ of the Gauss map $G$ can be expressed as

$$
\begin{aligned}
\Delta G= & \left(4+a^{2}\right) \alpha^{2} e_{1} \wedge e_{2}+(c+d) \alpha^{2} e_{1} \wedge e_{3}-(2 b+a d) \alpha^{2} e_{1} \wedge e_{4} \\
& +(2 b-a c) \alpha^{2} e_{2} \wedge e_{3}-(c+d) \alpha^{2} e_{2} \wedge e_{4} .
\end{aligned}
$$

We suppose that the flat surface $M$ with flat normal bundle has pointwise 1-type Gauss map. From (1.1) and (3.14), we get

$$
\begin{gathered}
\left(4+a^{2}\right) \alpha^{2}=f+f\left\langle C, e_{1} \wedge e_{2}\right\rangle \\
(c+d) \alpha^{2}=f\left\langle C, e_{1} \wedge e_{3}\right\rangle \\
(-2 b-a d) \alpha^{2}=f\left\langle C, e_{1} \wedge e_{4}\right\rangle \\
(2 b-a c) \alpha^{2}=f\left\langle C, e_{2} \wedge e_{3}\right\rangle \\
-(c+d) \alpha^{2}=f\left\langle C, e_{2} \wedge e_{4}\right\rangle
\end{gathered}
$$

Then, we have

$$
\left\langle C, e_{3} \wedge e_{4}\right\rangle=0
$$

By differentiating (3.20) with respect to $e_{1}$, we get

$$
\left\langle C, e_{1} \wedge e_{3}\right\rangle+\left\langle C, e_{2} \wedge e_{4}\right\rangle=0
$$

When we take the derivative of (3.20) with respect to $e_{2}$, we have

$$
\left\langle C, e_{1} \wedge e_{4}\right\rangle+\left\langle C, e_{2} \wedge e_{3}\right\rangle-a\left\langle C, e_{2} \wedge e_{4}\right\rangle=0
$$

If we evaluate the derivative of (3.22) with respect to $e_{2}$ again, we get

$$
\begin{aligned}
2\left\langle C, e_{1} \wedge e_{2}\right\rangle= & -(c+d)\left\langle C, e_{1} \wedge e_{3}\right\rangle+a c\left\langle C, e_{1} \wedge e_{4}\right\rangle \\
& +a d\left\langle C, e_{2} \wedge e_{3}\right\rangle+(c+d)\left\langle C, e_{2} \wedge e_{4}\right\rangle
\end{aligned}
$$

By using (3.15), (3.16), (3.17), (3.18), (3.19), (3.21) and (3.23) we then have

$$
f=\left(4+a^{2}+(c+d)^{2}+a b c+a^{2} c d-a b d\right) \alpha^{2}=A \alpha^{2}
$$

that is, a smooth function $f$ depends on $s$ and $t$. Differentiating (3.24) with respect to $e_{1}$, we have

$$
e_{1}(f)=-2 c A \alpha^{3} .
$$


On the other hand, by differentiating (3.19) with respect to $e_{1}$ and by using (3.12), (3.15), (3.17), (3.18), (3.19), (3.24) and (3.25) we obtain

$$
4 b^{2}+2 a b d-2 a b c-a^{2} c d-(c+d)^{2}=0 .
$$

By substituting (3.13) into (3.26) we get

$$
\left(k_{1}^{2}-k_{2}^{2}\right)\left(1+k_{1}^{2}+k_{2}^{2}+k_{1}^{2} k_{2}^{2}\right)=0
$$

and from (3.27) we obtain that $k_{1}= \pm k_{2}$. In particular, if we take as $k_{1}=k_{2}=$ 0 , we obtain Clifford torus. For the other cases, we obtain the tensor product surface of two logarithmic spirals.

Conversely, we assume that $k_{1}^{2}=k_{2}^{2}$. In that case the flat surface $M$ with flat normal bundle is given by the parametrization (3.8) has pointwise 1-type Gauss map for the function

$$
f(s, t)=\left(4+a^{2}+(c+d)^{2}+a b c+a^{2} c d-a b d\right) \alpha^{2}=A \alpha^{2}
$$

and the constant vector

$$
\begin{aligned}
C= & \frac{1}{A}\left(\left(4+a^{2}-A\right) e_{1} \wedge e_{2}+(c+d) e_{1} \wedge e_{3}-(2 b+a d) e_{1} \wedge e_{4}\right) \\
& +\frac{1}{A}\left((2 b-a c) e_{2} \wedge e_{3}-(c+d) e_{2} \wedge e_{4}\right) .
\end{aligned}
$$

Now, we assume that the flat surface $M$ with flat normal bundle is given by the parametrization (3.9). We research whether this surface has pointwise 1-type Gauss map. We can write as

$$
u(s)=c_{1}(\varepsilon \cos (2 s))^{-\frac{1}{2}},
$$

where if $\cos (2 s)>0($ resp. $<0)$, then $\varepsilon=1$ (resp. $=-1)$. Analogously, we can write as

$$
h(t)=c_{2}(\delta \cos (2 t))^{-\frac{1}{2}},
$$

where if $\cos (2 t)>0(<0$, respectively) then $\delta=1(-1$,respectively). By using (3.3), (3.4) and (3.5) we have components of the second fundamental form and the connection forms as:

$$
\begin{gathered}
h_{11}^{3}=0, \quad h_{12}^{3}=-\lambda(s, t), \quad h_{22}^{3}=\varkappa(s, t) \lambda(s, t) \\
h_{11}^{4}=-\lambda(s, t), \quad h_{12}^{4}=\varkappa(s, t) \lambda(s, t), \quad h_{22}^{4}=-\left(1+\varkappa^{2}(s, t)\right) \lambda(s, t)
\end{gathered}
$$

and

$$
\begin{aligned}
& \omega_{12}=\tau(s, t) \lambda(s, t) \omega_{1}+\beta(s, t) \lambda^{2}(s, t) \omega_{2} \\
& \omega_{34}=\tau(s, t) \lambda(s, t) \omega_{1}+\beta(s, t) \lambda^{2}(s, t) \omega_{2} .
\end{aligned}
$$


By covariant differentiation with respect to $e_{1}$ and $e_{2}$, we get

$$
\begin{aligned}
\tilde{\nabla}_{e_{1}} e_{1} & =\tau \lambda e_{2}-\lambda e_{4}, \\
\tilde{\nabla}_{e_{2}} e_{1} & =\beta \lambda^{2} e_{2}-\lambda e_{3}+\varkappa \lambda e_{4}, \\
\tilde{\nabla}_{e_{1}} e_{2} & =-\tau \lambda e_{1}-\lambda e_{3}+\varkappa \lambda e_{4}, \\
\tilde{\nabla}_{e_{2}} e_{2} & =-\beta \lambda^{2} e_{1}+\varkappa \lambda e_{3}-\left(1+\varkappa^{2}\right) \lambda e_{4}, \\
\tilde{\nabla}_{e_{1}} e_{3} & =\lambda e_{2}+\tau \lambda e_{4}, \\
\tilde{\nabla}_{e_{2}} e_{3} & =\lambda e_{1}-\varkappa \lambda e_{2}+\beta \lambda^{2} e_{4} \\
\tilde{\nabla}_{e_{1}} e_{4} & =\lambda e_{1}-\varkappa \lambda e_{2}-\tau \lambda e_{3}, \\
\tilde{\nabla}_{e_{2}} e_{4} & =-\varkappa \lambda e_{1}+\left(1+\varkappa^{2}\right) \lambda e_{2}-\beta \lambda^{2} e_{3},
\end{aligned}
$$

where

$$
\begin{aligned}
\varkappa(s, t) & =\frac{2(\varepsilon \sin (2 s))(\delta \sin (2 t))}{\left(1-(\varepsilon \sin (2 s))^{2}(\delta \sin (2 t))^{2}\right)^{\frac{1}{2}}}, \\
\tau(s, t) & =\frac{(\varepsilon \sin (2 s))(\delta \cos (2 t))}{(\varepsilon \cos (2 s))}, \\
\beta(s, t) & =\frac{c_{1} c_{2}(\delta \sin (2 t))\left((\varepsilon \cos (2 s))^{2}-(\varepsilon \sin (2 s))^{2}(\delta \cos (2 t))^{2}\right)}{(\varepsilon \cos (2 s))^{\frac{5}{2}}(\delta \cos (2 t))^{\frac{5}{2}}}, \\
\lambda(s, t) & =\frac{1}{W^{\frac{1}{2}}}=\frac{(\varepsilon \cos (2 s))^{\frac{3}{2}}(\delta \cos (2 t))^{\frac{3}{2}}}{c_{1} c_{2}\left(1-(\varepsilon \sin (2 s))^{2}(\delta \sin (2 t))^{2}\right)^{\frac{1}{2}}} .
\end{aligned}
$$

By using (2.1), straight-forward computation the Laplacian $\Delta G$ of the Gauss map $G$ can be expressed as

$$
\begin{aligned}
\Delta G= & \left(4+5 \varkappa^{2}+\varkappa^{4}\right) \lambda^{2} e_{1} \wedge e_{2}+\left(-e_{2}(\varkappa \lambda)-\beta\left(2+\varkappa^{2}\right) \lambda^{3}\right) e_{1} \wedge e_{3} \\
& +\left(e_{2}\left(\left(2+\varkappa^{2}\right) \lambda\right)-\beta \varkappa \lambda^{3}\right) e_{1} \wedge e_{4} \\
& +\left(e_{1}(\varkappa \lambda)+\tau\left(2+\varkappa^{2}\right) \lambda^{2}\right) e_{2} \wedge e_{3}+\left(-e_{1}\left(\left(2+\varkappa^{2}\right) \lambda\right)+\tau \varkappa \lambda^{2}\right) e_{2} \wedge e_{4} .
\end{aligned}
$$

We suppose that the flat surface $M$ with flat normal bundle has pointwise 1-type Gauss map. From (1.1) and (3.29), we get

$$
\begin{aligned}
& \left(4+5 \varkappa^{2}+\varkappa^{4}\right) \lambda^{2}=f+f\left\langle C, e_{1} \wedge e_{2}\right\rangle, \\
- & e_{2}(\varkappa \lambda)-\beta\left(2+\varkappa^{2}\right) \lambda^{3}=f\left\langle C, e_{1} \wedge e_{3}\right\rangle, \\
& e_{2}\left(\left(2+\varkappa^{2}\right) \lambda\right)-\beta \varkappa \lambda^{3}=f\left\langle C, e_{1} \wedge e_{4}\right\rangle, \\
& e_{1}(\varkappa \lambda)+\tau\left(2+\varkappa^{2}\right) \lambda^{2}=f\left\langle C, e_{2} \wedge e_{3}\right\rangle, \\
- & e_{1}\left(\left(2+\varkappa^{2}\right) \lambda\right)+\tau \varkappa \lambda^{2}=f\left\langle C, e_{2} \wedge e_{4}\right\rangle .
\end{aligned}
$$

Then we have

$$
\left\langle C, e_{3} \wedge e_{4}\right\rangle=0 .
$$


By differentiating (3.35) with respect to $e_{1}$, we get

$$
\left\langle C, e_{2} \wedge e_{4}\right\rangle-\left\langle C, e_{1} \wedge e_{3}\right\rangle+\varkappa\left\langle C, e_{2} \wedge e_{3}\right\rangle=0 .
$$

By considering together with (3.31), (3.33), (3.34) and (3.36), we have

$$
\begin{aligned}
-e_{1}\left(\left(2+\varkappa^{2}\right) \lambda\right)+\tau \varkappa \lambda^{2}+\varkappa & \left(e_{1}(\varkappa \lambda)+\tau\left(2+\varkappa^{2}\right) \lambda^{2}\right) \\
+ & e_{2}(\varkappa \lambda)+\beta\left(2+\varkappa^{2}\right) \lambda^{3}=0 .
\end{aligned}
$$

On the other hand, after some long computations we have

$$
\begin{gathered}
e_{1}(\varkappa)=\frac{4(\varepsilon \sin (2 s))(\varepsilon \cos (2 s))^{\frac{1}{2}}(\delta \cos (2 t))^{\frac{5}{2}}}{c_{1} c_{2}\left(1-(\varepsilon \sin (2 s))^{2}(\delta \sin (2 t))^{2}\right)^{\frac{3}{2}}}, \\
e_{2}(\varkappa)=\frac{4 \lambda(\varepsilon \cos (2 s))(\delta \sin (2 t))(\delta \cos (2 t))^{-1}}{\left(1-(\varepsilon \sin (2 s))^{2}(\delta \sin (2 t))^{2}\right)^{\frac{3}{2}}} \\
-\frac{4 \lambda(\varepsilon \sin (2 s))^{2}(\varepsilon \cos (2 s))^{-1}(\delta \sin (2 t))(\delta \cos (2 t))}{\left(1-(\varepsilon \sin (2 s))^{2}(\delta \sin (2 t))^{2}\right)^{\frac{3}{2}}}, \\
e_{1}(\lambda)=\frac{\left((\varepsilon \cos (2 s))^{2}(\delta \sin (2 t))(\delta \cos (2 t))^{2}\right)}{c_{1}^{2} c_{2}^{2}\left(1-(\varepsilon \sin (2 s))^{2}(\delta \sin (2 t))^{2}\right)^{\frac{3}{2}}}\left(-3+2(\varepsilon \sin (2 s))^{2}\right. \\
\left.+(\varepsilon \sin (2 s))^{2}(\delta \sin (2 t))^{2}\right)
\end{gathered}
$$

and

$$
\begin{aligned}
e_{2}(\lambda)= & \zeta\left(\left(-3+2(\delta \sin (2 t))^{2}+(\varepsilon \sin (2 s))^{2}(\delta \sin (2 t))^{2}\right)\right. \\
& \left.-(\delta \sin (2 t))^{2}\left(-3+2(\varepsilon \sin (2 s))^{2}+(\varepsilon \sin (2 s))^{2}(\delta \sin (2 t))^{2}\right)\right), \\
\zeta= & \frac{\lambda\left(\varepsilon \sin (2 s)(\varepsilon \cos (2 s))^{\frac{1}{2}}(\delta \cos (2 t))^{\frac{1}{2}}\right)}{c_{1} c_{2}\left(1-(\varepsilon \sin (2 s))^{2}(\delta \sin (2 t))^{2}\right)^{\frac{3}{2}}} .
\end{aligned}
$$

By combining (3.38), (3.39), (3.40) and (3.41) with (3.37), we obtain that this equation is not satisfied. So, there is no flat surface with flat normal bundle given by the parameterization (3.9) which has pointwise 1-type Gauss map.

Corollary 3.7. Let $M$ be flat surface with flat normal bundle given by the parameterization (3.2). $M$ has pointwise 1-type Gauss map of the first kind if and only if it is a Clifford Torus.

Proof. From Theorem 3.6 the flat surface $M$ with flat normal bundle is given by the parameterization (3.2) has pointwise 1-type Gauss map for the function

$$
f(s, t)=A \alpha^{2}
$$


and the constant vector

$$
\begin{aligned}
C= & \frac{1}{A}\left(\left(4+a^{2}-A\right) e_{1} \wedge e_{2}+(c+d) e_{1} \wedge e_{3}-(2 b+a d) e_{1} \wedge e_{4}\right) \\
& +\frac{1}{A}\left((2 b-a c) e_{2} \wedge e_{3}-(c+d) e_{2} \wedge e_{4}\right)
\end{aligned}
$$

with $k_{1}^{2}=k_{2}^{2}$, where

$$
A=\left(4+a^{2}+(c+d)^{2}+a b c+a^{2} c d-a b d\right) .
$$

We assume that the surface $M$ has pointwise 1-type Gauss map of the first kind. Then, we obtain $C=0$, that is, all components of $C$ is zero. Then, we get $k_{1}=k_{2}=0$. This completes the proof.

Theorem 3.8. An oriented minimal surface $M$ in the Euclidean space $\mathbb{E}^{4}$ has pointwise 1-type Gauss map of the first kind if and only if $M$ has a flat normal bundle [6].

Theorem 3.9. There exists no minimal surface given by the parameterization (3.2) with pointwise 1-type Gauss map of the first kind.

Proof. We suppose that the surface $M$ given by the parameterization (3.2) is minimal surface with pointwise 1-type Gauss map of the first kind. From Theorem 3.8 we have $R^{D}=0$. Since the surface $M$ is minimal and its normal bundle is flat then (2.2) and (2.4) imply, respectively

$$
\begin{gathered}
h_{11}^{3}+h_{22}^{3}=0 \text { and } h_{11}^{4}+h_{22}^{4}=0 \\
h_{12}^{3}\left(h_{11}^{4}-h_{22}^{4}\right)+h_{12}^{4}\left(h_{22}^{3}-h_{11}^{3}\right)=0 .
\end{gathered}
$$

By combining (3.3), (3.4), (3.42) and (3.43) we have

$$
h_{22}^{3}=h_{11}^{4}=h_{22}^{4}=0 .
$$

The equation (3.44) conflicts with the regularity of the surface.

Theorem 3.10. (See [6]). A non-planar minimal oriented surface $M$ in the Euclidean space $E^{4}$ has pointwise 1-type Gauss map of the second kind if and only if, with respect to some suitable local orthonormal frame $\left\{e_{1}, e_{2}, e_{3}, e_{4}\right\}$ on $M$, the shape operators of $M$ are given by

$$
A_{3}=\left(\begin{array}{cc}
\rho & 0 \\
0 & -\rho
\end{array}\right) \text { and } A_{4}=\left(\begin{array}{cc}
0 & \varepsilon \rho \\
\varepsilon \rho & 0
\end{array}\right)
$$

where $\varepsilon= \pm 1$ and $\rho$ is a smooth non-zero function on $M$.

Theorem 3.11. Let $M$ be minimal surface given by the parameterization (3.2). Then $M$ has pointwise 1-type Gauss map of the second kind if and only if it is parametrized by

$$
f(t, s)=\frac{b d}{\sqrt{|\cos (2 s+c)|}}(\cos s \cos t, \cos s \sin t, \sin s \cos t, \sin s \sin t)
$$


or

$$
f(t, s)=\frac{b d}{\sqrt{|\cos (2 t+c)|}}(\cos s \cos t, \cos s \sin t, \sin s \cos t, \sin s \sin t)
$$

where $b, d$ and $c$ are real constants.

Proof. We assume that $M$ is a minimal surface with pointwise 1-type Gauss map of second kind. In that case the mean curvature of $M$ is zero and we have

$$
h_{22}^{3}=0
$$

and

$$
h_{11}^{4}+h_{22}^{4}=0 .
$$

By using (2.5), the Laplacian $\Delta G$ of the Gauss map $G$ is written as

$$
\Delta G=\|h\|^{2} G+2 R^{D} e_{3} \wedge e_{4},
$$

where $R^{D} \neq 0$. In the opposite case, $M$ has pointwise 1-type Gauss map of the first kind. By using (2.4), (3.45) and (3.46) we get

$$
R^{D}=2 h_{12}^{3} h_{11}^{4} \neq 0 .
$$

Since $M$ has pointwise 1-type Gauss map of the second kind, from (1.1) and (3.47) we have

$$
\|h\|^{2} G+2 R^{D} e_{3} \wedge e_{4}=f G+f C
$$

for some smooth non-zero function $f$ on $M$ and some constant vector $C$. Since the vector $C$ is a linear combination of $e_{1} \wedge e_{2}, e_{1} \wedge e_{3}, e_{1} \wedge e_{4}, e_{2} \wedge e_{3}, e_{2} \wedge e_{4}$, $e_{3} \wedge e_{4}$. From (3.49) we get

$$
\begin{gathered}
\|h\|^{2}=f\left(1+\left\langle C, e_{1} \wedge e_{2}\right\rangle\right) \\
2 R^{D}=f\left\langle C, e_{3} \wedge e_{4}\right\rangle \neq 0
\end{gathered}
$$

and

$$
\left\langle C, e_{1} \wedge e_{3}\right\rangle=\left\langle C, e_{1} \wedge e_{4}\right\rangle=\left\langle C, e_{2} \wedge e_{3}\right\rangle=\left\langle C, e_{2} \wedge e_{4}\right\rangle=0
$$

Since $h_{12}^{3}$ is not equal to zero on $M$, it follows that $\|h\| \neq 0$ or $\left\langle C, e_{1} \wedge e_{2}\right\rangle \neq-1$. Differentiating $\left\langle C, e_{1} \wedge e_{3}\right\rangle=0$ with respect to $e_{1}$ and $e_{2}$, we get

$$
h_{12}^{3}\left\langle C, e_{1} \wedge e_{2}\right\rangle+h_{11}^{4}\left\langle C, e_{3} \wedge e_{4}\right\rangle=0
$$

and

$$
h_{12}^{4}\left\langle C, e_{3} \wedge e_{4}\right\rangle=0,
$$

respectively. On the other hand, differentiating $\left\langle C, e_{1} \wedge e_{4}\right\rangle=0$ with respect to $e_{1}$ and $e_{2}$, we have

$$
h_{12}^{4}\left\langle C, e_{1} \wedge e_{2}\right\rangle=0
$$

and

$$
h_{12}^{3}\left\langle C, e_{3} \wedge e_{4}\right\rangle+h_{11}^{4}\left\langle C, e_{1} \wedge e_{2}\right\rangle=0,
$$


respectively. The equation (3.54) implies that $h_{12}^{4}=0$ or $\left\langle C, e_{1} \wedge e_{2}\right\rangle=0$. If $\left\langle C, e_{1} \wedge e_{2}\right\rangle=0$ then from (3.55) we get $h_{12}^{3}\left\langle C, e_{3} \wedge e_{4}\right\rangle=0 . h_{12}^{3}$ is not equal to zero on $M$. Hence we have $\left\langle C, e_{3} \wedge e_{4}\right\rangle=0$ and (3.51) implies that $R^{D}=0$. This is a contradiction. So $\left\langle C, e_{1} \wedge e_{2}\right\rangle \neq 0$ and $h_{12}^{4}=0$. By using (3.52) and (3.55) we obtain

$$
\left(h_{12}^{3}\right)^{2}=\left(h_{11}^{4}\right)^{2} \text {. }
$$

From (3.45) and (3.3) we get $\dot{u}=0$ or $h^{\prime}=0$. Firstly we assume that $h^{\prime} \neq 0$. Then we have $u=d=$ constant. By considering together with (3.3), (3.4), (3.46) and (3.56) we obtain

$$
h(t)=\frac{c}{\sqrt{|\cos (2 t+b)|}} .
$$

Now we assume that $\dot{u} \neq 0$. Then we have $h=d=$ constant. By using (3.3) and (3.4) with $h=d$, we can see that (3.56) is satisfied directly. So, if we consider (3.4), (3.46) for $h=d$ we obtain

$$
u(s)=\frac{c}{\sqrt{|\cos (2 s+b)|}},
$$

where $b, c$ and $d$ are real constants.

If we consider as both $\dot{u}=0$ and $h^{\prime}=0$, then the surface $M$ is not minimal surface.

On the other hand by using (3.47), (3.48), (3.50), (3.51), (3.55) and (3.56) (or see the proof of Theorem 5 in $[6]$ ) we can find the function $f$ and the constant vector $C$ as

$$
f(s)=8\left(h_{12}^{3}\right)^{2}
$$

and

$$
C=-\frac{e_{1} \wedge e_{2}}{2}+\varepsilon \frac{e_{3} \wedge e_{4}}{2} .
$$

Hence the minimal surface $M$ has pointwise 1-type Gauss map of the second kind for the function $f$ and the constant vector $C$ given by (3.57) and (3.58), respectively. This completes the proof.

\section{REFERENCES}

[1] Arslan K., Bayram, B. K., Bulca, B., Kim, Y. H., Murathan, C., Öztürk, G., Rotational embeddings in $E^{4}$ with pointwise 1-type Gauss map, Turk. J. Math. 35 (2011), 493-499.

[2] Arslan K., Bayram, B. K., Bulca, B., Kim, Y. H., Murathan, C., Öztürk, G., Vranceanu surface in $E^{4}$ with pointwise 1-type Gauss map, Indian J. Pure. Appl. Math. 42 (2011), 41-51.

[3] Chen, B.Y., Piccinni, P., Submanifolds with finite type-Gauss map, Bull. Austral. Math. Soc., 35 (1987), 161-186. 
[4] Choi, M., Kim, Y.H., Characterization of the helicoid as ruled surfaces with pointwise 1-type Gauss map, Bull. Korean Math. Soc. 38 (2001), 753-761.

[5] Choi, M., Kim, D.S., Kim Y.H., Helicoidal surfaces with pointwise 1-type Gauss map, J. Korean Math. Soc. 46 (2009), 215-223.

[6] Dursun, U., Arsan, G.G., Surfaces in the Euclidean space $E^{4}$ with pointwise 1-type Gauss map, Hacet. J. Math. Stat. 40 (2011), 617-625.

[7] Dursun, U., Turgay, N.C., General rotational surfaces in Euclidean space $E^{4}$ with pointwise 1-type Gauss map, Math. Commun. 17 (2012), 71-81.

[8] Yoon, D.W., Some properties of the Clifford torus as rotation surface, Indian J. Pure. Appl. Math. 34 (2003), 907-915.

[9] Goemans W., Woestyne I.V., On flat tensor product surfaces, HogeschoolUniversiteit Brussel, Faculteit Economie en Management 17 (2011), 9 pages. 